\title{
MODELO PROCESUAL DEL ESTRÉS EN LA DISPEPSIA FUNCIONAL: IMPLICACIONES PARA LA EVALUACIÓN Y EL TRATAMIENTO
}

\author{
SERGIO TOBÓN ${ }^{1}$, STEFANO VINACCIA ${ }^{2}$ y BONIFACIO SANDÍN ${ }^{3}$ \\ ${ }^{1}$ Universidad de Antioquia, Colombia \\ ${ }^{2}$ Universidad de San Buenaventura, Colombia \\ ${ }^{3}$ Universidad Nacional de Educación a Distancia, España
}

(Aceptado en noviembre de 2003)

\begin{abstract}
La dispepsia funcional (DF) tiene una alta prevalencia en la población general y se ha asociado a factores psicológicos, sociales y biológicos. El presente artículo tiene como objetivo presentar un modelo explicativo de la DF a partir del modelo procesual del estrés. Acorde a éste, se presenta evidencia en torno a la implicación de los siguientes siete tipos de variables en esta condición médica: demandas psicosociales, evaluación cognitiva, respuestas de estrés, estrategias de afrontamiento, factores sociales, factores disposicionales y estatus de salud. Además, se tiene en cuenta el Helicobacter pylori y otros factores relevantes para este trastorno. Se concluye que la DF es un problema de salud asociado a factores biológicos, psicológicos y sociales. El modelo procesual del estrés posibilita comprender la interrelación de estos factores, ayuda a sistematizar las aportaciones de la investigación y orienta la contribución del psicólogo clínico en la evaluación y la terapia de este trastorno.
\end{abstract}

Palabras clave: Dispepsia funcional, dispepsia no ulcerosa, estrés, psicopatología, psicosomática, psicología y salud, evaluación, terapia psicológica.

The stress process model in the functional dyspepsia: Implications for its evaluation and treatment

Functional dyspepsia (FD) is very prevalent in the general population and has been associated with psychological, social and biological factors. The aim of this article is to present an explanation of FD from the stress process perspective. The paper shows the implication of seven types of variables in this medical condition, i.e.: psychosocial demands, cognitive evaluation, stress response, coping strategies, social factors, dispositional factors and health status. Also, it is considered the possible rol of the Helicobacter pylori and other relevant factors. We conclude that FD is a health problem associated with psychological, biological and social factors. The stress process model makes a possible way to understand the relationship among these factors, helps to systematize the research contribution and guides the clinical psychology in the evaluation and therapy.

Key words: Functional dyspepsia, non-ulcer dyspepsia, stress, psychopathology, psychosomatics, health psychology, evaluation, psychological therapy.

\section{INTRODUCCIÓN}

En la última década, la dispepsia funcional (DF) ha comenzado a despertar el interés de los psicólogos debido a la dificultad para ser explicada desde el modelo biomédico tradicional. Este problema de salud tiene una alta prevalencia en la población general (Talley, Zinsmeister, Schleck y Mel-

Correspondencia: Sergio Tobon, Cl. 21, 22-45 La

Ceja, Colombia. Correo-e: stobon1@netcard.net.net ton, 1992; Misiewicz, 1993), la cual puede situarse en un rango que va del $18 \%$ al $30 \%$ (Krag, 1982; Jones et al., 1990; Kurata, 1994; Quartero, Post, Numans, de Melker y de Wit, 1999; Talley et al., 2000; Hu et al., 2002; Li, Nie, Sha y Su, 2002).

La DF es un motivo frecuente de consulta médica en atención primaria (Jones et al., 1990), a pesar de que sólo consultan el $28 \%$ de las personas que tienen síntomas dispépticos (Jones y Lideard, 1989). 
Además, supone altos costes económicos en la utilización de los servicios de salud (Jones et al., 1990; Koloski, Talley y Boyce, 2001), en la medida que las personas con DF tienden a tener 2,6 veces más ausencias laborales por enfermedad que los controles (Nyren, Adami, Gustavsson y Loof, 1986). Por ejemplo, en Estados Unidos el coste relacionado con la DF ha sido estimado en más de 1,2 billones de dólares por año (Drossman et al., 1993).

La DF se refiere a dolor abdominal superior o malestar epigástrico recurrente o persistente frecuentemente asociado a síntomas tales como saciedad temprana, náuseas o vómito, en ausencia de una causa orgánica identificable por medios diagnósticos convencionales (Panganamamula, Fisher y Parkman, 2002; Talley et al., 2000; Danesh y Pounder, 2000), tales como métodos radiológicos, endoscópicos o histológicos. El diagnóstico de la DF implica, por lo tanto, descartar otras enfermedades del sistema digestivo que frecuentemente tienen síntomas similares tales como la úlcera péptica, la enfermedad por reflujo gastro-esofágico, el síndrome de colon irritable, y el cáncer gástrico, entre otras (Berstad et al. 2001; Talley et al. 2000; Tack et al. 2001).

La DF se diagnóstica cuando los síntomas están presentes durante doce semanas, no necesariamente consecutivas, y dentro de los doce meses precedentes, de acuerdo a los criterios de Roma II (Talley et al., 2000). De manera específica, el diagnóstico de la DF se basa en los siguientes criterios (Talley et al., 2000): (1) debe haber dolor y malestar centrado en el abdomen superior, de forma persistente y recurrente (2) los síntomas deben darse en ausencia de enfermedad orgánica que probablemente explique los síntomas (incluye evaluación endoscópica) (3) no debe haber evidencia de que los síntomas se alivien exclusivamente con la defecación o se asocien a cambios en la frecuencia o consistencia de las deposiciones; y (4) no deben darse síntomas predominantes de reflujo gastro-esofágico.

La DF se clasifica en los siguientes tipos, acorde a los criterios de Roma II (Talley et al., 2000):

(1) Dispepsia tipo úlcera: El síntoma predominante es dolor centrado en el abdomen superior.

(2) Dispepsia tipo dismotilidad: El síntoma predominante es la sensación de malestar centrado en el abdomen superior; esta sensación puede estar caracterizada por o asociada con distensión abdominal superior, saciedad temprana, malestar en el hemiabdomen superior, eructos o náusea.

(3) Dispepsia inespecífica: Este tipo se da cuando los síntomas de dispepsia no presentan un predominio correspondiente a alguno de los tipos anteriores.

La DF reviste gran interés para los psicólogos clínicos debido a que es una condición médica asociada estrechamente a factores psicosociales (Hui, Shiu y Lam, 1991; Folks y Kinney, 1992; Pauli, Herschbach, Weiner y von-Rad, 1992; Kellner, 1994; Haug, 2002a; Tobón, Vinaccia y Sandín, 2003a). Además, muchos trastornos psicopatológicos se acompañan de síntomas propios de esta enfermedad (Read, 2002). Por otra parte, cada vez es más frecuente la recomendación de practicar evaluación y terapia psicológica en aquellas personas con DF y problemas de salud mental (Tobón, Vinaccia y Sandín, 2003b). Todo ello justifica la necesidad de que el psicólogo clínico conozca en detalle todos los mecanismos psicológicos, sociales y de intervención en esta enfermedad funcional, acorde a las más recientes investigaciones en el campo.

Es por ello que el propósito del presente artículo consiste en presentar un modelo sobre la relación de diversos factores psicosociales con la DF que posibiliten una clara 
orientación en torno a la evaluación, diagnóstico y tratamiento de esta condición médica, así como la implementación de futuras investigaciones en este campo. Para cumplir con este fin, se ha seguido el modelo procesual del estrés desarrollado por Sandín $(1995,1999)$, el cual relaciona el estrés psicosocial con la salud. Luego se analiza la relación entre el Helicobacter pylori ( $H$. pylori) y los factores psicológicos en la DF tipo úlcera y se presenta un modelo hipotético. Finalmente, se exponen una serie de planteamientos en torno a la evaluación y el tratamiento de la DF desde el campo de la Psicología Clínica.

\section{MODELO PROCESUAL DEL ESTRÉS Y DISPEPSIA FUNCIONAL}

El estrés implica una perturbación del funcionamiento habitual del individuo, el cual puede ser entendido en términos biológicos (homeostasis) o psicológicos (rutina, bienestar percibido, estado emocional), cuya alteración es generada por algún tipo de estimulación (externa o interna) que suele denominarse estresor (estímulo, suceso, pensamiento, idea, etc.) (Sandín, 1999). El modelo procesual del estrés asume el estrés como una ausencia de equilibrio entre las demandas del contexto y los recursos que posee la persona para afrontarlas. En el caso de la DF se propone el modelo expuesto en la Figura 1. Para ello se tiene como base el modelo de Sandín (1999) quien describe la relación entre el estrés y la salud a partir de siete etapas interdependientes: demandas psicosociales, evaluación cognitiva, respuesta de estrés, estrategias de afrontamiento, variables sociales, variables disposicionales y estatus de salud.

1. Demandas psicosociales 2. Evaluación cognitiva
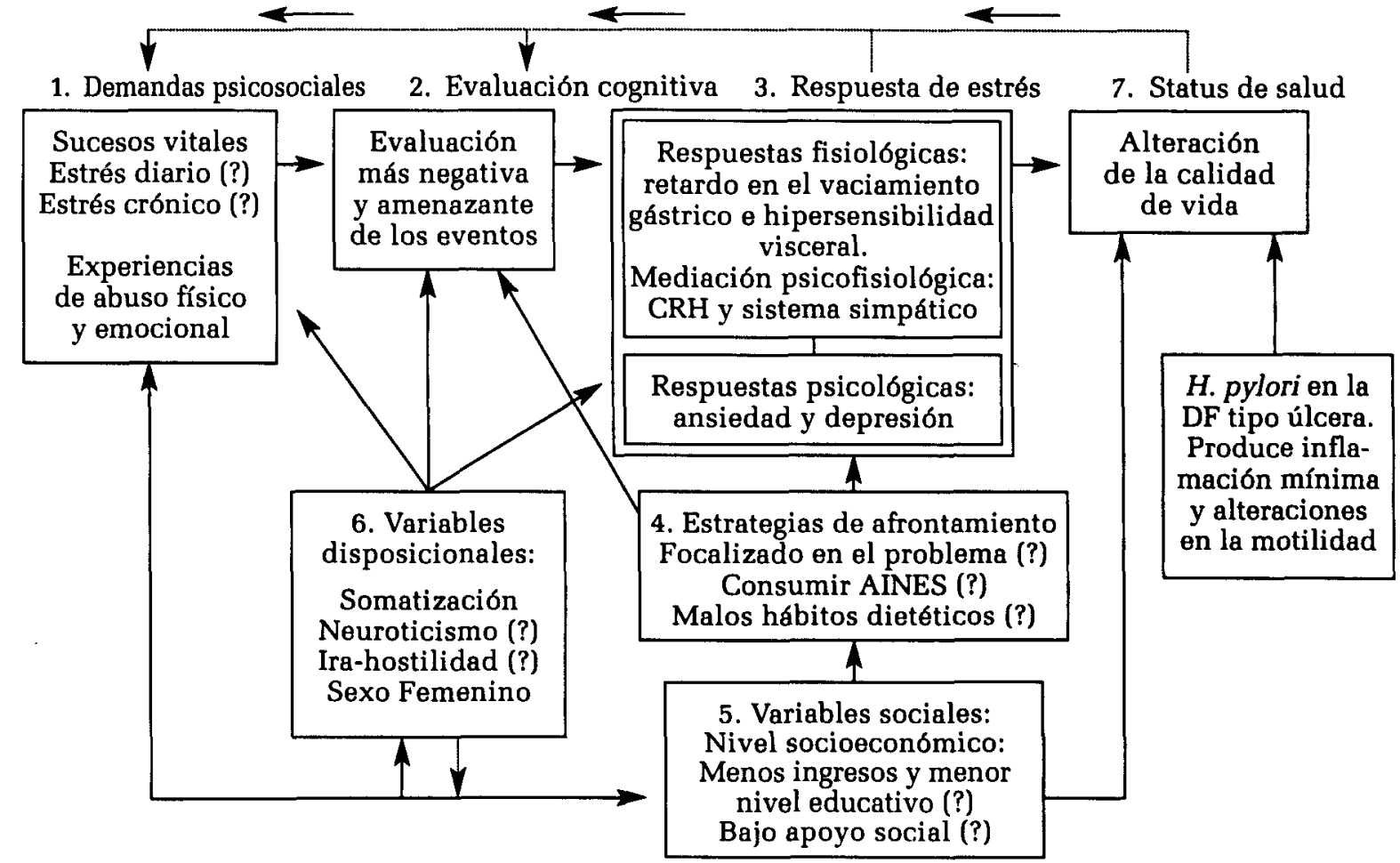

3. Respuesta de estrés

7. Status de salud

Figura 1. Modelo procesual del estrés y dispepsia funcional. Basado en Sandín (1999). Se indican las principales relaciones entre los componentes.

Nota: El signo «?» significa que falta evidencia concluyente. DF = dispepsia funcional; $C R H=$ corticotrophin releasing factor. AINES = antiinflamatorios no esteroideos. En línea discontinua se indican relaciones de feedback. 


\section{Demandas psicosociales}

Dadas unas determinadas condiciones sociales, el inicio o exacerbación de las enfermedades se relaciona, con frecuencia, con determinadas demandas psicosociales que implican para la persona cambios en su proceso normal de adaptación. Tales demandas psicosociales se clasifican en tres clases: Sucesos vitales (situaciones extraordinarias que demandan cambios significativos en el patrón normal de adaptación), estrés diario (sucesos cotidianos relacionados con satisfacciones o contrariedades) y estrés crónico (son situaciones de tensión crónica que se mantienen en el tiempo y se relacionan con áreas tales como la laboral, la familiar y la sociocultural).

En la DF, diversos estudios han reportado la implicación de eventos vitales en esta condición médica, en comparación con personas sanas (Tobón et al., 2003a; Haug et al., 1995; Lau et al., 1996). Lee et al. (2000) compararon 30 personas DF con 30 adultos sanos que visitaron una clínica hepatobiliar para la evaluación médica por quejas no orgánicas, sin DF. Se evaluó la presencia de eventos vitales percibidos mediante autoinforme y se encontró que las personas con DF presentaron un mayor número de tales eventos. Este estudio reviste especial importancia porque hubo control de variables tales como el rol de enfermo, los síntomas de enfermedad y la consulta.

Aparte de los sucesos vitales, el estrés diario se ha relacionado con los síntomas de enfermedades gastrointestinales tales como el síndrome de intestino irritable y la enfermedad de Crohn (Sandín, Chorot y Santed, 1999), pero son escasos los estudios de este factor en la DF. Hui et al. (1991) halló una mayor percepción negativa de contrariedades diarias en pacientes con DF que en controles, pero un estudio posterior no hallo tal evidencia (Lau et al, 1996), ni tampoco una mayor frecuencia de eventos diarios.
Finalmente, las personas con DF tienden a informar altos grados de estrés ocupacional, familiar y preocupaciones económicas (Medias y Rutle, 1993). En un reciente estudio epidemiológico, más de la mitad de las personas diagnosticadas con DF y malestar epigástrico juzgaron que habían tenido una situación de estrés crónico y asociaron el estrés con sus síntomas (Allescher et al., 1999).

Complementariamente a lo anterior, la evidencia científica muestra que las situaciones de abuso físico y sexual son frecuentes en personas con trastornos gastrointestinales funcionales, dentro de los cuales está la dispepsia funcional (Drossman et al., 1990; Talley et al., 1994). Esta asociación ha sido apoyada por un estudio reciente de tipo poblacional donde se encontró una relación significativa de la DF con situaciones de abuso (Li et al., 2002).

El estrés parece ser uno de los factores que influyen para que las personas con DF busquen ayuda (Koloski et al, 2001). $\mathrm{Al}$ respecto, Lydeard y Jones (1989) encontraron que los sucesos vitales estresantes son más experimentados por personas que acuden a consultas médicas, aunque no está claro el rol del apoyo social ni de las estrategias de afrontamiento en la búsqueda de atención en los servicios de salud por parte de las personas con DF (Koloski et al, 2001).

\section{Evaluación cognitiva}

El impacto estresante de los diversos agentes estresores y su influencia en el inicio, curso o exacerbación de los síntomas depende, en gran medida, de cómo sean éstos evaluados y percibidos por cada persona (Sandín, 1995). Tal evaluación se lleva a cabo en dos fases: evaluación automática (es una evaluación primaria que cataloga la demanda psicosocial como aceptable o amenazante, activándose el organismo en una u otra dirección) y evaluación controlada (consiste 
en el análisis de las demandas psicosociales y en los recursos que se poseen para afrontarlas, así como de las acciones a seguir para responder a la situación). La respuesta de estrés se produce cuando la persona percibe que no tiene los recursos personales necesarios para afrontarla. Acorde a lo anterior, se ha indicado que las personas con DF tienden a percibir de una forma más negativa y amenazante los sucesos vitales en comparación con sujetos sanos (Bennett, Beaurepaire, Langeluddecke, Kellow y Tennant, 1991; Hui, Ho y Lam, 1991; Hernández, Arandia y Dehesa, 1993; Lau et al., 1996; Lee et al., 2000). Se puede hipotetizar que, como consecuencia de la evaluación más negativa y amenazante de los sucesos vitales, estos sobrepasan los recursos de las personas para manejarlos y se produce la respuesta de estrés, tanto en el plano psicológico como físico. Tales respuestas de estrés influirían de forma directa o indirecta en el inicio o evolución de la DF (o en sus síntomas).

\section{Respuestas de estrés}

Una vez la persona percibe que las demandas ambientales sobrepasan la capacidad de respuesta, se ponen en acción dos procesos interrelacionados: activación de emociones negativas (por ejemplo, ansiedad y depresión) y activación fisiológica a partir del sistema neuroendocrino y sistema nervioso autónomo.

Las emociones negativas producen cambios en el funcionamiento gastrointestinal. En este sentido hay evidencia sólida y consistente de la asociación de la ansiedad y la depresión con la DF, lo cual constituye una señal de su posible papel en el origen o curso de esta enfermedad.

La depresión se ha evaluado en la DF empleando por lo general el conocido cuestionario de autoinforme Symptom Checklist-90-Revised (SCL-90) y el BDI
(Beck Depresión Inventory). En general se ha concluido que las personas con DF tienen mayores niveles de depresión que las personas sanas o controles con otras enfermedades (véase la Tabla 1). La depresión no sólo se asocia con la DF, sino también con ciertos síntomas característicos de esta condición médica, tales como la sensación de llenura (Chou et al., 2001) y náuseas (Chou et al., 2001; Handa et al., 1999).

La ansiedad se ha tendido a evaluar de manera conjunta con la depresión. Varios estudios han encontrado una asociación consistente de esta emoción negativa con la DF (véase la Tabla 2). La ansiedad también se ha hallado asociada a los síntomas dispépticos en la población general (Hu et al., 2002) y en muestras clínicas (Maganti, Ebert y Jones, 2002). Esto ha llevado a proponer que la ansiedad influencia la presencia y gravedad de los síntomas (Li et al., 2002).

En la DF, la depresión se ha asociado a menor apoyo social (Lee et al., 2000). Esto corrobora lo propuesto por Aneshendsel y Stone (1982) en el sentido de que los bajos niveles de apoyo social contribuyen a la manifestación de los síntomas depresivos.

En relación con las respuestas fisiológicas, los estudios recientes aportan evidencia sobre dos anormalidades fundamentales en la DF: (1) retardo en el vaciamiento gástrico, y (2) la hipersensibilidad visceral (Haug, 2002). Con respecto a la primera anormalidad, se ha constatado que ante situaciones de estrés agudo la motilidad gastrointestinal sufre cambios, siendo la inhibición del vaciamiento gástrico el patrón más consistente (Monnikes et al., 2001). Esto es apoyado por estudios realizados en personas sanas donde los estresores de laboratorio retardan el vaciamiento gástrico (Camilleri et al., 1984; Thompson et al., 1983). Con respecto a la hipersensibilidad, el estrés podría estar implicado disminuyendo el 
Tabla 1. Descripción de algunos estudios que han analizado la relación entre depresión y DF

\begin{tabular}{llllll}
\hline Estudio & Tipo de estudio & $n$ & Grupo control & Instrumento & Conclusiones \\
\hline Hu et al., 2002 & $\begin{array}{l}\text { Estudio poblacional } \\
\text { de prevalencia } \\
\text { (China) }\end{array}$ & 1649 & $\begin{array}{l}\text { Escala de Ansiedad } \\
\text { y Depresión } \\
\text { Hospitalaria }\end{array}$ & $\begin{array}{l}\text {-Asociación de la depresión } \\
\text { con la DF. } \\
\text {-No hubo asociación de la } \\
\text { depresión con la búsqueda } \\
\text { de atención médica. }\end{array}$ \\
Li et al., 2002 & $\begin{array}{l}\text { Estudio } \\
\text { epidemiológico } \\
\text { (China) }\end{array}$ & 72 & $\begin{array}{l}\text { con pacientes } \\
\text { congánica }\end{array}$ & $\begin{array}{l}\text { Escala } \\
\text { de Depresión } \\
\text { de Zung }\end{array}$ & $\begin{array}{l}\text { Mayor depresión } \\
\text { en las personas con DF en } \\
\text { comparación con los grupos } \\
\text { de control. }\end{array}$ \\
& $\begin{array}{l}\text {-197 pacientes } \\
\text { con otras } \\
\text { enfermedades }\end{array}$ & & \\
& & &
\end{tabular}

$\begin{array}{lllll}\text { Jiang et al., } & \begin{array}{l}\text { Estudio transversal } \\ \text { (China) }\end{array} & 56 & 56 \text { personas sanas } & \text { SCL-90 }\end{array}$

Chou et al., Estudio comparativo $39 \quad$ Escala de Depresión 2001 de Zung

Mayor depresión en personas con DF.

-Los pacientes con DF y depresión tuvieron una mayor frecuencia de síntomas que los pacientes con DF no deprimidos. - No hubo correlación entre el tipo específico de anormalidad electrogastrográfica y la presencia o ausencia de depresión.

$\begin{array}{llll}\begin{array}{l}\text { Norton et al., } \\ 1999\end{array} & \text { Estudio de prevalencia } & 29 & 98 \text { personas } \\ & \text { y transversal } & \text { sin DF } \\ & \text { en estudiantes } & \\ & \text { universitarios (Canadá) }\end{array}$

Lee et al. 2000 Estudio transversal
(Sur Corea) 30

30 personas sanas que visitaron una clínica hepatobiliar

Haug et al., 1995
Estudio transversal
(Haukeland)

100

-100 personas $\quad$ Inventario de
sanas
-100 personas
con úlcera duodenal

Inventario de
Depresión de Beck

Inventario de Depresión de Beck

Mayor depresión en los estu diantes con DF en compara ción con los estudiantes $\sin \mathrm{DF}$.

Mayor nivel de depresión en las personas con DF.

Mayor depresión en las personas con DF en comparación con las personas con úlcera duodenal y personas sanas. umbral para la percepción del malestar o aumentando la percepción de la distensión gástrica a partir de la activación simpática (Iovino, Azpiroz y Domingo, 1995). Por su parte, Monnikes et al. (2001) han planteado que el estrés incrementa la sensibilidad ante la estimulación visceral experimental, si el estresor induce un cambio emocional significativo.

Los mecanismos psicofisiológicos a través de los cuales el estrés induce alteraciones en la motilidad y la sensibilidad visceral todavía son poco claros. Algunos estudios muestran cómo la hormona liberadora de la corticotropina (CRH) y la hormona liberadora de la tirotropina (TRH) afectan la regulación vagal del estómago (Tache, Garric y Rayboud, 1990; Weiner, 1996), y tales hormonas pueden activarse en presencia del estrés (Sandín, 1999). Así mismo, la CRH también puede inducir hipersensibilidad visceral en presencia del estrés, tal como ha sido sugerido por estudios experimenta- 
Tabla 2. Descripción de algunos estudios sobre ansiedad y DF

\begin{tabular}{|c|c|c|c|c|c|}
\hline Estudio & Tipo de estudio & $n$ & Grupo control & Instrumento & Conclusiones \\
\hline Hu et al., 2002 & $\begin{array}{l}\text { Estudio poblacional } \\
\text { de prevalencia } \\
\text { (China) }\end{array}$ & 303 & 1346 & $\begin{array}{l}\text { Escala de Ansiedad } \\
\text { Hospitalaria }\end{array}$ & $\begin{array}{l}\text { - Mayor ansiedad } \\
\text { en las personas con DF } \\
\text { en comparación } \\
\text { con las personas sanas. }\end{array}$ \\
\hline Li et al., 2002 & $\begin{array}{l}\text { Estudio } \\
\text { epidemiológico } \\
\text { y retrospectivo } \\
\text { (China) }\end{array}$ & 72 & $\begin{array}{l}\text {-84 pacientes } \\
\text { con dispepsia } \\
\text { orgánica } \\
-197 \text { pacientes } \\
\text { con otras } \\
\text { enfermedades }\end{array}$ & $\begin{array}{l}\text { Escala de Ansiedad } \\
\text { (SAS) }\end{array}$ & $\begin{array}{l}\text { Mayor grado de ansiedad } \\
\text { en las personas con DF } \\
\text { que en los grupos de control. }\end{array}$ \\
\hline $\begin{array}{l}\text { Jiang et al., } \\
2000\end{array}$ & $\begin{array}{l}\text { Estudio transversal } \\
\text { (China) }\end{array}$ & 56 & 56 personas sanas & SCL-90 & $\begin{array}{l}\text { Mayor grado de ansiedad } \\
\text { en las personas con DF } \\
\text { en comparación con el grupo } \\
\text { control. }\end{array}$ \\
\hline $\begin{array}{l}\text { Norton et al., } \\
1999\end{array}$ & $\begin{array}{l}\text { Estudio } \\
\text { de prevalencia } \\
\text { y transversal } \\
\text { en estudiantes } \\
\text { universitarios } \\
\text { (Canadá) }\end{array}$ & 29 & $\begin{array}{l}98 \text { personas } \\
\sin D F\end{array}$ & $\begin{array}{l}\text {-Inventario } \\
\text { de Ansiedad } \\
\text { de Beck } \\
\text { - Indice de } \\
\text { Sensibilidad } \\
\text { a la Ansiedad }\end{array}$ & $\begin{array}{l}\text { Los estudiantes con DF } \\
\text { presentaron mayor ansiedad } \\
\text { que los estudiantes sanos. }\end{array}$ \\
\hline $\begin{array}{l}\text { Lee et al., } \\
2000\end{array}$ & $\begin{array}{l}\text { Estudio transversal } \\
\text { (Sur Corea) }\end{array}$ & 30 & $\begin{array}{l}30 \text { personas sanas } \\
\text { que visitaron } \\
\text { una clínica } \\
\text { hepatobiliar }\end{array}$ & $\begin{array}{l}\text {-Inventario de } \\
\text { Ansiedad Estado- } \\
\text { Rasgo } \\
\text {-SCL-90 }\end{array}$ & $\begin{array}{l}\text {-No hubo diferencias } \\
\text { en ansiedad estado-rasgo } \\
\text { entre personas con DF } \\
\text { y el grupo control. } \\
\text { - Hubo mayor nivel de } \\
\text { ansiedad medida con el } \\
\text { SCL-90 en las personas } \\
\text { con DF con respecto al grupo } \\
\text { control. }\end{array}$ \\
\hline $\begin{array}{l}\text { Haug et al., } \\
1995\end{array}$ & $\begin{array}{l}\text { Estudio transversal } \\
\text { (Haukeland) }\end{array}$ & 100 & $\begin{array}{l}-100 \text { personas } \\
\text { sanas } \\
-100 \text { personas con } \\
\text { úlcera duodenal }\end{array}$ & $\begin{array}{l}\text { Escala de Ansiedad } \\
\text { Estado-Rasgo }\end{array}$ & $\begin{array}{l}\text { Mayor nivel de } \\
\text { ansiedad en las personas con } \\
\text { DF en comparación con los } \\
\text { grupos de control. }\end{array}$ \\
\hline
\end{tabular}

les (Monnikes et al., 2001). En una línea diferente, pero complementaria, los factores psicológicos pueden estar asociados con la alteración de la motilidad mediante la supresión del tono vagal (Haug, Svebak, Hausken, Wilhelmsen et al., 1994).

\section{Estrategias de afrontamiento}

Las estrategias de afrontamiento son acciones conductuales y cognitivas que aplican las personas para hacer frente a las demandas estresantes, a la activación fisiologica y al malestar emocional asociado a la experiencia del estrés (Sandín, 1995, 1999). Las estrategias de afrontamiento pueden dirigirse al problema, con el fin de eliminar o reducir las fuentes que producen el estrés; también pueden estar dirigidas a la reducción de los estados emocionales negativos y de la activación fisiológica. Cuando el afrontamiento no 
logra disminuir el proceso del estrés, entonces hay una alta probabilidad de que se produzcan alteraciones en la salud.

Es posible que el empleo de estrategias inadecuadas de afrontamiento del estrés lleve a que éste se mantenga en el tiempo sin resolverse, incidiendo en el origen o exacerbación de la DF. En apoyo de este planteamiento se encuentra un estudio reciente realizado por Lee et al. (2000). Estos investigadores examinaron las estrategias de afrontamiento en un grupo de 30 pacientes con DF, comparándolo con un grupo de control de 30 personas sanas. Hallaron que, en comparación con las personas sanas, los pacientes con DF tendían a utilizar un menor grado de estrategias de afrontamiento focalizadas en el problema. No hubo diferencias significativas en el empleo de otras estrategias de afrontamiento. El resultado de este estudio sugiere que las personas con DF tienden a (1) estar menos orientadas a definir el problema (2) generar menos alternativas de solución (3) estar menos orientadas a evaluar las alternativas en términos de coste-beneficio, y cambiar de actuación acorde a esto, y (4) estar menos orientadas a obtener bienestar emocional e información de otros. Se requieren nuevos estudios para contrastar estos hallazgos y llegar a conclusiones sólidas.

El estrés puede ser afrontado mediante conductas perjudiciales para la salud tales como consumir alcohol y fumar. En el tratamiento de la DF se ha sugerido eliminar la conducta de fumar y disminuir el consumo de licor, café y AINES (drogas antiinflamatorias no esteroideas) (véase, por ejemplo, Feinle-Bisset y Andrews, 2003; Panganamamula, Fisher y Parkman, 2002). Sin embargo, a pesar de las creencias médicas y populares, estos factores tienen una baja contribución al origen y exacerbación de los síntomas en la DF (Richter, 1991).

Lo anterior se ha corroborado en varias investigaciones. Talley, Weaver y Zins- meister (1994) no hallaron asociación entre el alcohol y la DF. Este mismo resultado fue obtenido por Archimandritis, Sipsas, Tryphonos, Tsirantonaki y Tjivras (1995) en un estudio prospectivo realizado en Grecia. Además del alcohol, no hallaron asociación entre la DF y factores tales como fumar y consumir café y bebidas gaseosas. En otro estudio reciente, el alcohol y el tabaco tampoco fueron asociados con un mayor riesgo de DF (Holtmann, Gschossmann, Holtmann y Talley, 2001). Con respecto a las AINES, Talley et al. (1994) no hallaron evidencia de asociación entre el consumo de aspirina y acetaminofén con la DF. Sin embargo, un estudio reciente sí halló asociación de la DF frecuente con el consumo de AINES (Holtmann et al., 2001). Así mismo, recientemente se halló que los malos hábitos dietéticos fueron un factor de riesgo para la DF (Li et al., 2002), pero esto requiere de nuevos estudios.

El bajo grado de asociación de conductas tales como fumar y consumir alcohol con la DF no significa que estas no tengan implicación en esta enfermedad. Debido a que tales conductas tienden a agravar o exacerbar los síntomas dispépticos, es posible que las personas tiendan a disminuirlas una vez los comienzan a experimentar y esta podría ser la causa de la baja asociación con la DF. En apoyo de esto está el hecho de que la mayoría de estudios en esta área han sido retrospectivos y no evalúan periodos suficientemente amplios para determinar una posible relación con el inicio de la enfermedad.

\section{Factores sociales}

El entorno social está implicado en las demandas psicosociales, las respuestas del estrés, su afrontamiento y la condición de salud. Dentro de esta dimensión son de especial relevancia el nivel socioeconómico y el apoyo social. El primero inclu- 
ye el nivel de ingresos, el nivel educativo y el nivel ocupacional, factores asociados al proceso de salud en el plano de la morbilidad y mortalidad y están implicados en la depresión, la autoestima y la indefensión (Sandín, 1999). El segundo se vincula al estatus socioeconómico y tiene efectos amortiguadores del estrés, así como efectos directos sobre la salud.

Las personas con DF tienden a tener menores ingresos (Haug et al., 1994), así como una menor satisfacción con éstos (Li et al, 2002). En este último estudio, también se refiere que las personas con DF tienden a tener un pobre ambiente social, insatisfacción con la comunidad y presión de la sociedad. Igualmente, se ha hallado que las personas con DF tienden a tener un menor nivel educativo respecto a las personas sanas.

En respuesta a las situaciones de estrés, las personas con bajo apoyo social son más vulnerables a enfermar y a experimentar perturbaciones emocionales que los que tienen apoyo social elevado (DeLongis, Folkman y Lazarus, 1988). En un estudio reciente sobre la relación entre el estrés y la DF, Lee et al. (2000) examinaron el apoyo social en un grupo de pacientes con DF, comparándolo con un grupo control de personas sanas. Encontraron que, en comparación con los sujetos sanos, los pacientes con DF tuvieron menos apoyo interpersonal que el grupo control. Este factor requiere ser estudiado en futuros estudios con el fin de tener evidencia más sólida y contrastada.

\section{Variables disposicionales}

Son factores inherentes a la persona que tienen la capacidad de modular las primeras cuatro etapas del estrés. Aquí están incluidas las dimensiones de personalidad, los tipos de reacción al estrés, los factores hereditarios, el sexo y la raza. El estrés tiene mayores consecuen- cias negativas sobre la salud cuando hay rasgos de personalidad «tóxicos», tales como el neuroticismo o la ira-hostilidad. En el caso de la DF, se ha hallado la implicación de estos factores en varios estudios, así como también la del factor sexo.

En la infancia, determinadas condiciones familiares y sociales (actitudes de la familia frente a las manifestaciones digestivas o la enfermedad, modelamiento de determinadas formas de reacción frente al estrés, etc.) pueden afectar el desarrollo psicosocial, aumentando así la susceptibilidad al estrés, disminuyendo el apoyo social y desarrollando rasgos de personalidad negativos como la somatización y el neuroticismo. Así mismo, la familia puede contribuir a desarrollar DF reforzando síntomas digestivos o sirviendo de modelo en el aprendizaje de conductas anormales de enfermedad (Whitehead, 1996), lo cual está en la base del rasgo de somatización altamente asociado a la DF. Complementariamente, los modelos en la infancia en que se maximiza la relevancia del dolor abdominal pueden afectar las habilidades de afrontamiento (Richter, 1991) e incidir en el desarrollo de estilos negativos de reacción al estrés.

La somatización se ha definido como una manifestación somática del malestar psicológico; como una tendencia a manifestar síntomas somáticos, generalmente en personas con elevados niveles de ansiedad o depresión, en ausencia de una evidencia objetiva de enfermedad (Whitehead, 1996). Al respecto, los pacientes con DF informan más síntomas somáticos de tipo no gastrointestinal que la población general (Wilhelmsen, 2002). Esto ha sido corroborado por diversos estudios donde se ha hallado una asociación significativa entre la somatización y la DF mediante el empleo del cuestionario SCL-90 y grupos de control con personas sanas (Jonsson, Theorell y Gott- 
hard, 1995; Jorgensen et al., 1996; Lee et al., 2000).

Los síntomas de somatización pueden reflejar un modo cultural de expresión del estrés en ciertas poblaciones (Lee et al., 2000). En este sentido, se ha podido constatar cómo en ciertas comunidades el modo predominante de comunicar el malestar emocional es mediante terminología somática, como por ejemplo en Corea, donde estar enfermo de la mente o tener emociones negativas es equivalente a tener sensaciones anormales en la región epigástrica. En este país las expresiones de malestar físico son más comúnmente aceptadas que las expresiones de malestar emocional (Kim, 1999). Así mismo, está el hecho de que los chinos y japoneses informan más quejas gastrointestinales relacionadas con la DF (indigestion, peor apetito y gas) que otros grupos culturales (Marsella et al., 1973).

Muchos autores han informado que en los pacientes con DF existe una tendencia general neurótica o ansiosa (Camilleri, Malagelada y Kao, 1986; Talley et al., 1986, 1990; Bennett et al., 1991; Piubello et al., 1994). En un estudio reciente, Tanum y Malt (2001) evaluaron el rasgo de neuroticismo en personas con trastornos gastrointestinales funcionales (DF o síndrome de intestino irritable) mediante los cuestionarios EPQ (Eysenck Personality Questionnaire) y NEO-PI (NEO Personality Inventory). Entre los diferentes rasgos de personalidad que evalúan estos dos cuestionarios, sólo el neuroticismo se encontraba elevado en los pacientes con trastornos funcionales, comparado con los sujetos de control (participantes sanos). Los autores concluyen que el neuroticismo puede ser considerado como un marcador de vulnerabilidad hacia los dos tipos de trastornos gastrointestinales funcionales. Téngase en cuenta que los dos trastornos se ubican en el sistema digestivo y se caracterizan por ser fun- cionales y no están asociados a alteraciones estructurales.

En el caso del rasgo de ira-hostilidad, en una serie de estudios clásicos se observó de manera directa a través de fístulas gástricas la asociación de la ira con el incremento de la actividad ácido-péptica, la demora en el vaciamiento gástrico y el aumento en la motilidad (Beaumont, 1833; Wolf y Wolff, 1943; Engel, Reichsman y Segal, 1956). En esta misma línea, Bennett et al. (1992) hallaron una demora en el vaciamiento gástrico de líquidos y sólidos en personas con DF asociada a intentos de reprimir la ira. Langeluddecke et al., (1990) informa que las personas con DF son más hostiles que aquellas con enfermedades orgánicas. También se ha hallado que los problemas interpersonales en los cuales la ira no es expresada, se relacionaban con dispepsia (Stockton, Weinman y McColl, 1985). Un resultado similar es referido por Tanun y Malt (2001). Estos investigadores aplicaron el cuestionario BDHI (Buss-Durkee Hostility Inventory) a una muestra de pacientes con trastornos gastrointestinales funcionales (incluida la DF y el síndrome del colon irritable) y hallaron puntuaciones más elevadas en agresión encubierta que los sujetos sanos (grupo de control).

Con respecto al sexo, se ha indicado que la DF tiene una mayor prevalencia en las mujeres (Lee et al., 2000; Drossman et al., 1982). Es factible que el sexo femenino tenga mayores probabilidades de tener esta condición médica a causa de mayor sensibilidad a los síntomas, mayores probabilidades de abuso físico y mayor tolerancia de la sociedad a las quejas, que podría llevar a una mayor consulta. Es por ello que cuando el estudio se hace en un contexto poblacional, no se encuentran diferencias significativas entre hombres y mujeres (Li et al., 2002). Nuevos estudios son necesarios para contrastar esta hipótesis. 


\section{Estatus de salud}

La calidad de vida relacionada con la salud es el resultado de cómo se manejen las situaciones de estrés y se prevengan todos aquellos factores que afectan a la salud. En el caso de la DF, se ha podido constatar que las personas con esta enfermedad tienen una peor calidad de vida (Berstad et al., 2001), lo cual se evidencia en sus dificultades sociales y laborales (Hu et al., 2002). Incluso, dicha calidad de vida es peor que la de personas con enfermedades gastrointestinales estructurales (Wilhelmsen, 1995; Talley, Weaber y Zinsmeister, 1995).

\section{Helicobacter pylori (H. pylori)}

La $H$. pylori es una bacteria que se encuentra en la mucosa del estomago y produce una inflamación mínima asintomática en más de la mitad de la población mundial. Los estudios en esta área han constatado una fuerte relación etiológica entre esta bacteria y la úlcera péptica (duodenal o gástrica) (Haug, 2002; Carella et al., 1999; Gisbert, Boixeda y Martín de Argila, 1996a), y es por ello que se ha propuesto también como un factor de riesgo para la DF (Gisbert, Boixeda y Martín de Argila, 1996b). Sin embargo, las investigaciones son todavía controvertidas, aunque se ha hallado asociación de la bacteria con la DF tipo úlcera (Carella et al., 1999). En este último caso, el malestar en el abdomen superior sería el resultado de la gastritis histológica asociada a la bacteria junto con alteraciones fisiológicas inducidas por las toxinas (Carella et al., 1999). Sin embargo, no todos los pacientes con DF tipo úlcera están infectados.

En los pacientes DF infectados con $H$. pylori, nosotros hipotetizamos un modelo de causalidad basado en la interacción entre factores sociales, psicológicos y la bacteria (Figura 2). Hay evidencia concluyente de que las personas con bajas condiciones socioeconómicas tienen mayores probabilidades de ser infectadas por el $H$. pylori debido a peores condiciones higiénicas. Esto es un factor asociado, a la vez, a menor apoyo social y a mayores problemas psicológicos. Los factores psicológicos podrían interactuar con el $H$. pylori a partir de varios mecanismos. Uno podría ser a nivel inmunológico. Se ha descubierto que las personas con una respuesta inmune más intensa frente al $H$. pylori (altas concentraciones de anticuerpos IgG en suero) tienen mayores probabilidades de tener DF (Holtmann et al., 2001). Los factores psicológicos están asociados a cambios en la inmunocompetencia y, de esta forma, podrían favorecer una mayor patogenicidad de la bacteria. En segundo lugar, las alteraciones fisiológicas asociadas a la bacteria podrian complementarse con cambios en la función gastroduodenal asociados a situaciones de continuo estrés (p.ej., retardo en el vaciamiento gástrico o hipersensibilidad) y de esta forma producir los síntomas de dispepsia. Un tercer mecanismo, podría ser a través de conductas inadecuadas de afrontamiento del estrés tales como fumar y consumir alcohol y AINES, las cuales, aunque no están claramente asociadas con la etiología y curso de la DF, podrían desempeñar algún papel en un subgrupo de pacientes. Los efectos "agresivos» de tales conductas, sumados a la gastritis crónica histológica, inducirían malestar en el abdomen superior. Este es sólo un modelo hipotético y se requiere de futuros estudios que lo confirmen o refuten.

\section{EVALUACIÓN Y TRATAMIENTO PSICOLÓGICO}

La creciente evidencia en torno a la interrelación de diferentes factores psicosociales en la DF apoya incluir dentro de la 


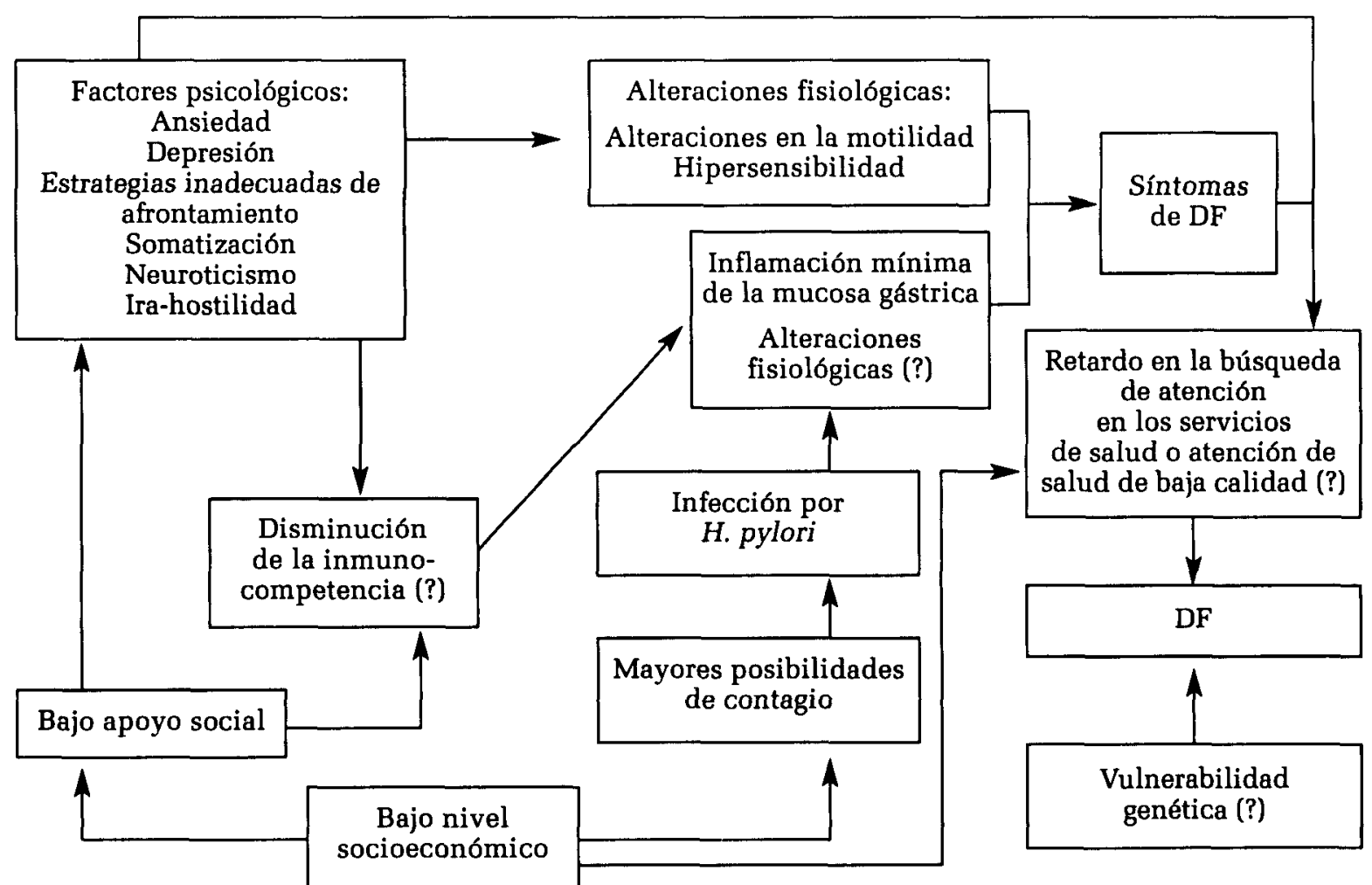

Figura 2. Modelo hipotético de interacción entre el $H$. pylori, los factores sociales y los factores psicológicos en el origen y curso de la DF tipo úlcera.

Nota: El signo «?» significa que falta evidencia concluyente.

intervención médica de esta enfermedad, la evaluación y el tratamiento psicológico, con el fin de incidir positivamente en la curación de los síntomas y mejorar la calidad de vida, tal como has sido sugerido recientemente (Tack et al., 2001; Tobón, Vinaccia y Sandín, 2003b).

\section{Evaluación psicológica}

El psicólogo clínico puede hacer sus aportaciones a la evaluación psicológica de los pacientes con dispepsia funcional, sobre todo en aquellos casos donde se sospeche de estrés. Para ello puede orientarse en el modelo procesual del estrés, considerando el análisis de las demandas psicosociales a las cuales están expuestos los pacientes, los patrones de evaluación cognitiva, las estrategias de afrontamiento, las respuestas de estrés en el ámbito psicológico y físico, los factores sociales implicados, las variables disposicionales y el estatus de salud relacionado con la calidad de vida, la consulta médica y las actitudes hacia el tratamiento.

De acuerdo a las experiencias previas de los pacientes y su situación particular, el psicólogo clínico debe establecer prioridades en la evaluación de estos factores, considerando los que sean más significativos para el diagnóstico integral y para el tratamiento. Por lo general es central evaluar las conductas tales como el consumo de alcohol, tabaco y AINES, así como el estado emocional, las estrategias de afrontamiento y la calidad de vida.

\section{Tratamiento psicológico}

Se ha constatado de forma general que la terapia psicológica tiene efectos positivos 
sobre los síntomas dispépticos y el malestar emocional (Tobón et al., 2003b). De manera más específica, a continuación se describen las modalidades de terapia psicológica que han tenido efectos positivos sobre los síntomas asociados a la DF: terapia de retroalimentación (Giles, 1976), manejo del estrés mediante técnicas conductuales (Uhes, Sweet y Cowles, 1989), terapia cognitiva (Haug, Svebak, Wilhelmsen, Berstad y Ursin, 1994), terapia cognitivo-conductual sola y combinada con tratamiento farmacológico (Vinaccia, Tobón, Martínez-Sánchez y Sandín, 2002), terapia hipnótica (Calvert, Houghton, Cooper, Morris y Whorwell, 2002), xiaoyu decoction (XYD) (una modalidad de intervención médica china combinada con psicoterapia) (Jiang, Lin y Zhang, 2000), terapia interpersonal psicodinámica breve (Hamilton, Guthrie, Credo, Thompson y Tomenson, 2000), y la group counseling psychotherapy (basada en la asesoría e intervención grupal) (Poitras et al., 2002).

Es esencial esclarecer el momento en el cual ha de aplicarse la terapia psicológica. Algunos autores recomiendan el empleo de ésta sólo cuando el tratamiento farmacologico falla y hay presencia de trastornos psicopatológicos (Tack et al., 2001; Tarilonte, 2002). Sin embargo, es factible que el empleo de la terapia psicológica desde el comienzo mejore de forma significativa el pronóstico y contribuya a una mejor calidad de vida en muchos pacientes. El tratamiento psicológico en la DF está indicado en aquellos casos donde hay altos niveles de estrés y puede darse en complemento con el tratamiento farmacológico desde el comienzo de la intervención (Vinaccia et al., 2002).

Hacia el futuro se pueden plantear al menos los siguientes retos (véase Tobón et al., 2003b): (1) replicar los estudios realizados con el fin de obtener evidencia concluyente; (2) incluir periodos de seguimiento mayores a un año; (3) determinar qué tipo de personas pueden beneficiarse de la terapia psicológica en la práctica clínica cotidiana; (4) abordar conjuntamente la modificación de conductas tales como el consumo de alcohol, de café y de tabaco; (5) determinar el coste de la intervención psicológica respecto de sus beneficios en la fase post-tratamiento en aspectos tales como consumo de medicamentos, visitas al médico o gastroenterólogo, número de admisiones al hospital, etc.; (6) comparar la efectividad de las diferentes modalidades de terapia psicológica solas y combinadas con la terapia farmacológica; (7) establecer la efectividad de la terapia psicológica teniendo en cuenta los diferentes tipos de DF; y (8) considerar los efectos de la terapia psicológica no sólo sobre los síntomas de la DF y alteraciones psicológicas, sino también sobre la calidad de vida del paciente.

\section{CONCLUSIONES}

El modelo procesual del estrés propuesto por Sandín $(1995,1999)$ es un enfoque heurístico que posibilita integrar la evidencia aportada por la investigación respecto a la asociación de diversos factores psicológicos, sociales y biológicos con DF. Este modelo, además, permite establecer relaciones entre variables y formular hipotesis para el desarrollo de nuevas investigaciones en el campo.

Acorde a la revision realizada desde el modelo procesual del estrés, la DF es un problema de salud asociado a los siguientes factores: demandas psicosociales (sucesos vitales), evaluación cognitiva (evaluación negativa y amenazante de los sucesos vitales), emociones negativas (ansiedad y depresión), variables moduladoras (somatización y sexo femenino) y estatus de salud (baja calidad de vida). Se requieren nuevos estudios con respecto a 
factores tales como el estrés diario y crónico, las estrategias de afrontamiento, el neuroticismo, la ira-hostilidad, el apoyo social y las condiciones socioeconómicas. Así mismo, es necesario determinar cómo se relacionan las variables psicosociales entre sí y con respecto al $H$. pylori dentro del marco de estudios prospectivos, teniendo en cuenta los diversos subtipos de la DF.

La evidencia recogida apoya el empleo de la evaluación y la terapia psicológica en el tratamiento de la DF en aquellos casos refractarios al tratamiento convencional o donde hay altos niveles de estrés. En este último caso la evaluación y la terapia pueden aplicarse desde el comienzo, de forma complementaria a la intervención médica. Sin embargo, se requieren nuevos estudios que brinden evidencia concluyente en torno a cuál modalidad de terapia psicológica es más efectiva y cual es la relación de costebeneficio en este tipo de intervención.

\section{REFERENCIAS BIBLIOGRÁFICAS}

Allescher, H.D., Adler, G., Hartung, J., Manns, M.P., Riemann, J.F., Wienbeck, M., y Classen, M. (1999). [Estudio Epidemiológico Prospectivo de Malestar Epigástrico (PRESTO). Ground work and preliminary results]. Deutsche Medizinische Wochenschrift, 124, 443-450.

Aneshendsel, C.S., y Stone, J.D. (1982). Stress and depression: a test of the buffering model of social support. Archives of General Psychiatry, 39, 1392-1396.

Archimandritis, A., Sipsas, N., Tryphonos, M., Tsirantonaki, M., y Tjivras, M. (1995). Significance of various factors in patients with functional dyspepsia and peptic ulcer disease in Greece. A comparative prospective study. Annales de Médecine Interne (París), 146, 299-303.

Beaumont, W. (1833). Experiments and observations on the gastric juice and the physiology of digestion. Plattsburg: FP Allen.
Bennett, E., Beaurepaire, J., Langeluddecke, P.M., Kellow, J.E., y Tennant, C.C. (1991). Life stress and non-ulcer dyspepsia, a case control study. Journal of Psychosomatic Research, 35, 579-590.

Bennett, E.J., Kellow, J.E., Cowan, H., Scott, A.M., Shuter, B., Langeluddecke, P.M., Hoschl, R., Jones, M.P., y Tennant, C.C. (1992). Suppression of anger and gastric emptying in patients with functional dyspepsia. Scandinavian Journal of Gastroenterology, 27, 869-874.

Berstad, A., Olafsson, S., Tefera, S., Hatlebakk, J.G., Gilja, O.H., y Hausken, T. (2001). Controversies in dyspepsia. The European Journal of Surgery Supplement, 586, 4-11.

Calvert, E.L., Houghton, L.A., Cooper, P., Morris, J., y Whorwell, P.J. (2002). Longterm improvement in functional dyspepsia using hypnotherapy. Gastroenterology, 12, 1778-1785.

Camilleri, M., Malagelada, J.R., y Kao, P.C. (1984). Influence of somatovisceral reflexes and selective dermatonal stimulation on postcibal antral pressure activity in man. The American Journal of Physiology, 247, G703-708.

Camilleri, M., Malagelada, J.R., y Kao, P.C. (1986). Gastric and autonomic responses to stress in functional dyspepsia. Digestive Diseases and Sciences, 31, 1169-1170.

Carella, A.M., Bianco, G., D'Alessandro, V., Villella, M., D’Amico, G., Mazzoccoli, G., Sperandeo, M., Annese, M.A., y Sabella, G. (1999). Dispepsia y Helicobacter pylori. Clin. Ter., 150, 67-76.

Chou, L.T., Wu, C.Y., Chen, H.P., Chang, C.S., Wong, P.G., Ko, C.W., y Chen, G.H. (2001). The correlation of depression and gastric dysrhythmia in functional dyspepsia. Journal of Clinical Gastroenterology, 33, 127-131.

Danesh, J., y Pounder, R.E. (2000). Eradication of Helicobacter pylori and non-ulcer dyspepsia. The Lancet, 355, 766-767.

Delongis, A., Folkman, S., y Lazarus, R.S. (1988). The impact of daily stress on health and mood: psychological and social resources as mediators. Journal of Personality and Social Psychology, 54, 486-495.

Drossman, D.A., Leserman, J., Nachman, G., Gluck, H., Toomey, T.C., y Mitchel, C.M. (1990). Sexual and physical abuse in 
women with functional or organic gastrointestinal disorders. Annals of Internal Medicine, 113, 828-833.

Drossman, D.A., Li, A., Andruzzi, E., Temple, R.D., Talley, N.J., Thompson, W.G., Whitehead, W.E., Janssens, J., Funch-Jensen, P., Corazziari, E., Richter, J.E., y Koch, G.G. (1993). U.S. Householder survey of functional gastrointestinal disorders: prevalence, sociodemography and health impact. Digestive Diseases and Sciences, 38, 1569-1580.

Drossman, D.A., Sandler, R.S., McKee, D.C., y Lovitz, A.J. (1982). Bowel patterns among subjects not seeking health care. Use of a questionnaire to identify a population with bowel dysfunction. Gastroenterology, 83, 529-534.

Engel, G.L., Reichsman, F., y Segal, H.L. (1956). A study of an infant with a gastric fistula. Behavior and the rate of total $\mathrm{Hcl}$ secretion. Psychosomatic Medicine, 18, 374-398.

Feinle-Bisset, C., y Andrews, J. M. (2003). Treatment of Functional Dyspepsia. Current Treatment Options in Gastroenterology, 6, 289-297.

Folks, D.G., y Kinney, C.K. (1992). The role of psychological factors in gastrointestinal conditions, a review pertinent to DSM-IV. Psychosomatics, 33, 257-270.

Giles, S.L. (1976). Separate and combined effects of biofeedback training and brief individual psychotherapy in the treatment of gastrointestinal disorders. Dissertation Abstract Int, 39, 2495B.

Gisbert, J.P., Boixeda, D., y Martín de Argila, C. (1996a). Infección por Helicobacter pylori y úlcera péptica. En D. Boixeda, J.P. Gisbert y C. Martín de Argila (Eds.), Infección por h.pylori ¿Dónde esta el limite? (pp. 135-157). Barcelona: Prous Science.

Gisbert, J.P., Boixeda, D., y Martín de Argila, C. (1996b). Infección por Helicobacter pylori y dispepsia no ulcerosa. En D. Boixeda, J.P. Gisbert y C. Martín de Argila (Eds.), Infección por $h$. pylori ¿Dónde esta el limite? (pp. 159-177). Barcelona: Prous Science.

Hamilton, J., Guthrie, E., Credo, F., Thompson, D., y Tomenson, B. (2000). Why should psychotherapy be a useful approach to management of patients with non ulcer dyspepsia? Gastroenterology, 119, 661-669.

Handa, M., Mine, K., Yamamoto, H., Tsutsui, S., Hayashi, H., Kinukawa, N., y Kubo, C. (1999). Esophageal motility and psychiatric factors in functional dyspepsia patients with or without pain. Digestive Diseases and Sciences, 44, 2094-2098.

Haug, T.T. (2002). [Dispepsia funcional - Una enfermedad psicosomática]. Tidsskrift for den Norske Laegeforening, 122, 1218-1222. (Original en Noruego).

Haug, T.T., Svebak, S., Hausken, T., Wilhelmsen, I., Berstad, A., y Ursin, H. (1994). Low vagal activity as mediating mechanism for the relationship between personality factors and gastric symptoms in functional dyspepsia. Psychosomatic Medicine, 56, 181-186.

Haug, T.T., Svebak, S., Wilhelmsen, I., Berstad, A., y Ursin, H. (1994). Psychological factors and somatic symptoms in functional dyspepsia. A comparison with duodenal ulcer and healthy controls. Journal of Psychosomatic Research, 38, 281-291.

Haug, T.T., Wilhelmsen, I., Berstad, A., y Ursin, H. (1995). Life events and stress in patients with functional dyspepsia compared with patients with duodenal ulcer and healthy controls. Scandinavian Journal of Gastroenterology, 30, 524-530.

Haug, T.T., Wilhelmsen, I., Svebak, S., Berstad, A., y Ursin, H. (1994). Psychotherapy in functional dyspepsia: A comparison with duodenal ulcer and healthy controls. Journal of Psychosomatic Research, 38, 735-744.

Hernández, D.E., Arandia, D., y Dehesa, M. (1993). Role of psychosomatic factors in peptic ulcer disease. Journal of Physiology, Paris, 87, 223-227.

Holtmann, G., Gschossmann, J., Holtmann, M., y Talley, N.J. (2001). H. pylori and functional dyspepsia: increased serum antibodies as an independent risk factor? Digestive Diseases and Sciences, 46, 1550-1557.

Hu, W.H., Wong, W.M., Lam, C.L., Lam, K.F., Hui, W.M., Lai, K.C., Xia, H.X., Lam, S.K., y Wong, B.C. (2002). Anxiety but not depression determines health care-seeking behaviour in Chinese patients with dyspepsia and irritable bowel syndrome: a 
population-based study. Alimentary Pharmacology? Therapeutics, 16, 2081-2088.

Hui, W.M., Ho, J., y Lam, S.K. (1991). Pathogenetic role of helicobacter pylori in duodenal ulcer disease. Multivariate analysis of factors affecting relapse. Digestive Diseases and Sciences, 36, 424-430.

Hui, W.M., Shiu, L.P., y Lam, S.K. (1991). A biopsychosocial model for functional dyspepsia. Gastroenterology, 101, 866-868.

Iovino, P., Azpiroz, F., y Domingo, E. (1995). The sympathetic nervous system modulates perception and reflex responses to gut distension in humans. Gastroenterology, 108, 680-686.

Jiang, B., Lin, J., y Zhang, Y. (2000). [Observación clínica de los síntomas mentales en la dispepsia funcioanal y su tratamiento]. Zhongguo Zhong Xi Yi Jie He Za Zhi Zhongguo Zhongxiyi Jiehe Zazhi, 20, 424426. [Original en Chino]

Jones, R., y Lydeard, S. (1989). Prevalence of symptoms of dyspepsia in the community. British Medical Journal, 298, 30-32.

Jones, R.H., Lydeard, S.T., Hobbs, F.D.R., Kenkre, J.E., Williams, E.I., Jones, S.J., Repper, J.A., Caldow, J.L., Dunwoodie, W.M., y Bottomley, J.M. (1990). Dyspepsia in England and Scotland. Gut, 31, 401-405.

Jonsson, B.H., Theorell, T., y Gotthard, R. (1995). Symptoms and personality in patients with chronic functional dyspepsia. Journal of Psychosomatic Research, 39, 93-102.

Jorgensen, L.S., Christiansen, P.M., Raundahl, U., y Ostgaard, S.E. (1996). Long- lasting functional abdominal pain and duodenal ulcer are associated with stress, vulnerability and symptoms of psychological stress. A controlled study including healthy and patient controls. Danish Medical Bulletin, 43, 359-363.

Kellner, R. (1994). Psychosomatic syndromes, somatization and somatoform disorders. Psychotherapy and Psychosomatics, 61, 4-24.

Kim, K. (1999). Culture and illness behaviors in South Korea. Transcultural Psychiatry, 36, 65-77.

Koloski, N.A., Talley, N.J., y Boyce, P.M. (2001). Predictors of health care seeking for irritable bowel syndrome and nonulcer dyspepsia: A critical review of the literature on symptom and psychosocial factors. The American Journal of Gastroenterology, 96, 1340-1349.

Krag, E. (1982). Non-ulcer dyspepsia introduction: Epidemiological data. Scandinavian Journal of Gastroenterology, 17, 6-8.

Kurata, J.H. (1994). Gastritis/nonulcer dyspepsia. En J.E. Everhart (Ed.), Digestive disease in the U.S.: epidemiology and impact (p. 409). Washington, DC: National Institutes of Health, National Institute of Diabetes, Digestive and Kidney Diseases Publication.

Langeluddecke, P., Goulston, K., y Tennant, C. (1990). Psychological factors in dyspepsia of unknown cause: A comparison with peptic ulcer disease. Journal of Psychosomatic Research, 34, 215-222.

Lau, G.K., Hui, W.M., y Lam, S.K. (1996). Life events and daily hassles in patients with atypical chest pain. The American Journal of Gastroenterology, 91, 2157-2162.

Lee, S.Y., Park, M.C., Choi, S.C., Nah, Y.H., Abbey, S.E., y Rodin, G. (2000). Stress, coping, and depression in non-ulcer dyspepsia patients. Journal of Psychosomatic Research, 49, 93-99.

Li, Y., Nie, Y., Sha, W., y Su, H. (2002). The link between psychosocial factors and functional dyspepsia: an epidemiological study. Chinese Medical Journal (Engl.), 115, 1082-1084.

Maganti, K., Ebert, C.C., y Jones, M.P. (2002). Predictive value of symptoms (Sxs) in functional dyspepsia (FD). American Journal of Gastroenterology, 97, Suppl.1, S41.

Marsella, A.J., Kinzie, D., y Gordon, P. (1973). Ethnic variations in the expression of depression. Journal of Cross-Cultural Psychology, 4, 435-459.

Medias, I.B., y Rutle, O. (1993). [Dispepsia y estilo de vida] Dyspepsi og livsstil. Tidsskrift for den Norske Laegeforening, 113, 947-950.

Misiewicz, J.J. (1993). Dyspepsia. En M.H. Sleisenger y J.S. Fordtran (Eds.), Gastrointestinal Disease (pp. 486-508). Philadelphia: WB Saunders.

Monnikes, H., Tebbe, J.J., Hildebrandt, M., Arck, P., Osmanoglou, E., Rose, M., Klapp, B., Wiedenmann, B., y Heymann-Monnikes, I. (2001). Role of stress in functional 
gastrointestinal disorders. Evidence for stress-induced alterations in gastrointestinal motility and sensitivity. Digestive Diseases, 19, 201-211.

Norton, G.R., Norton, P.J., Asmundson, G.J.G., Thomson, L.A., y Larsen, D.K. (1999). Neurotic butterflies in my stomach: The role of anxiety, anxiety sensitivity and depression in functional gastrointestinal disorders. Journal of Psychosomatic Research, 47, 233-240.

Nyren, O., Adami, H.O., Gustavsson, S., y Loof, L. (1986). Excess sick -listing in nonulcer dyspepsia. Journal of Clinical Gastroenterology, 8, 339-345.

Panganamamula, K.V., Fisher, R.S., Parkman, H.P. (2002). Functional (nonulcer) dyspepsia. Current Treatment Options in Gastroenterology, 5, 153-160.

Pauli, P., Herschbach, P., Weiner, H., y vonRad, M. (1992). [Factores psicológicos en la dispepsia no ulcerosa] Psychologische Faktoren der Non-ulcer dyspepsia (NUD). Psyhotherapie, Psychosomatik, Medizinisch Psychologie, 42, 295-301.

Piubello, W., Aimo, G.P., Superti, G., Raggi, G.C., Pistoso, S., Max, G., y Romanini, M. (1994). Caratteristiche psicologiche della dispepsia: Studio controllato con followup gastroscopico. Minerva Gastroenterologica e Dietologica, 40, 27-30.

Poitras, M.R., Verrier, P., So, C., Paquet, S., Bouin, M., y Poitras, P. (2002). Group counselling psychotherapy for patients with functional gastrointestinal disorders: development of new measures for symptom severity and quality of life. Digestive Diseases and Sciences, 47, 1297-307.

Quartero, A.O., Post, M.W., Numans, M.E., de Melker, R.A., y de Wit, N.J. (1999). What makes the dyspeptic patient feel ill? A cross sectional survey of functional health status, Helicobacter pylori infection, and psychological distress in dyspeptic patients in general practice. Gut, 45, 15-19.

Read, N.W. (2002). Food and hypersensitivity in functional dyspepsia. Gut, 51, 50-53.

Richter, J.E. (1991). Stress and psychological and environmental factors in functional dyspepsia. Scandinavian Journal of Gastroenterology, 182, 40-46.

Sandín, B. (1995). El estrés. En A. Belloch, B. Sandín y F. Ramos (Eds.), Manual de Psi- copatología (Vol. II, pp. 3-52). Madrid: McGraw-Hill.

Sandín, B. (1999) (Ed.). El estrés psicosocial: Conceptos y consecuencias clínicas. Madrid: Klinik.

Sandín, B., Chorot, P., y Santed, M.A. (1999). El estrés diario. En B. Sandín (Ed.), El estrés psicosocial: Conceptos y consecuencias clínicas (pp. 81-99). Madrid: Klinik.

Stockton M, Weinman J., y McColl I. (1985). An investigation of psychosocial factors in patients with upper abdominal pain: a comparison with other groups of surgical outpatients. Journal of Psychosomatic Research, 29, 191-198.

Tache, Y., Garric, T., y Raybould, H. (1990). Central nervous system action of peptides to influence gastrointestinal motor function. Gastroenterology, 98, 517-528.

Tack, J., Bisschops, R., y DeMarchi, B. (2001). Causes and treatment of functional dyspepsia. Current Gastroenterology Reports, 3, 503-508.

Talley, N.J., Fett, S.L., Zinsmeister, A.R., Melton, L.J. 3rd (1994). Gastrointestinal tract symptoms and self-reported abuse: a population-based study. Gastroenterology. 107, 1040-1049.

Talley, N.J., Fung, L.H., Gilligan, I.J., McNeil, D., y Piper, D.W. (1986). Association of anxiety, neuroticism, and depression with dyspepsia of unknown cause: a case-control study. Gastroenterology, 90, 886-892.

Talley, N.J., Phillips, S.F., Bruce, B., Twomey, C.K., Zinsmeister, A.R., y Melton, L.J. (1990). Relation among personality and symptoms in non-ulcer dyspepsia and the irritable bowel syndrome. Gastroenterology, 99, 327-333.

Talley, N.J., Stanghellini, V., Heading, R.C., Koch, K.L., Malagelada, J.R., y Tytgat, G.N. (2000). Functional gastroduodenal disorders. En D.A. Drossman, E. Corazziari, N.J. Talley, W. Grant Thompson y W.E. Whitehead (Eds.), Rome II: The functional gastrointestinal disorders ( $2^{\text {nd }}$ ed.). Mclean, VA: Degnon Associates.

Talley, N.J., Weaber, A.L., y Zinsmeister, A.R. (1995). Impact of dyspepsia on quality of life. Digestive Diseases and Sciences, 40 , 584-589. 
Talley, N.J., Weaver, A.L., y Zinsmeister, A.R. (1994). Smoking, alcohol, and nonsteroidal anti inflammatory drugs in outpatients with functional dyspepsia and among dyspepsia subgroups. American Journal of Gastroenterology, 89, 524-528.

Talley, N.J., Zinsmeister, A.R., Schleck, C.D., y Melton, L.J.3 ${ }^{\text {rd. }}$ (1992). Dyspepsia and dyspepsia subgroups: a population-based study, Gastroenterology, 102, 1259-1268.

Tanum, L., y Malt, U.F. (2001). Personality and physical symptoms in nonpsychiatric patients with functional gastrointestinal disorder. Journal of Psychosomatic Research, 50, 139-146.

Tarilonte, M.A. (2002). Dispepsia en atención primaria. Revista Andaluza de Patología Digestiva, 25, 193-199.

Thompson, D.G., Richelson, E., y Malagelada, J.R. (1983). Perturbation of upper gastrointestinal function by cold stress. Gut, 24, 277-283.

Tobón, S., Vinaccia, S., y Sandín, B. (2003a). Estrés psicosocial y factores psicológicos en la dispepsia funcional. Anales de Psicología, 2, 223-234.

Tobón, S., Vinaccia, S., y Sandín, B. (2003b). Tratamiento psicológico de la dispepsia funcional: un análisis crítico. Terapia Psicológica, 21, 173-182.
Uhes, M.J., Sweet, A.A., y Cowles, S. (1989). A behaviourally orientated stress reduction program and its effects upon self-reported gastric complaints. Stress, 5, 57-61

Vinaccia, S., Tobón, S., Sandín, B., y Martínez-Sánchez, F. (2002). Efectos de un programa psicoterapéutico, combinado a una terapia farmacológica en el tratamiento del distrés psicológico y la sintomatología física de sujetos con dispepsia no ulcerosa (DNU). Revista Colombiana de Gastroenterología, 16, 187-192.

Weiner, H. (1996).Use of animal models in peptic ulcer disease. Psychosomatic Medicine, 58, 524-545.

Whitehead, W.E. (1996). Psychosocial aspects of functional gastrointestinal disorders. Gastroenterology Clinics of North America, 25, 21-34

Wilhelmsen, I. (1995). Quality of life in upper gastrointestinal disorders. Scandinavian Journal of Gastroenterology (Supplement), 211, 21-25.

Wilhelmsen, I. (2002). Somatization, sensitization, and functional dyspepsia. Scandinavian Journal of Psychology, 43, 177-80.

Wolf, S., y Wolff, H.G. (1943). Human Gastric Function. New York: Oxford University Press. 